\title{
DER ZIPFEL DES DIPLOMATISCHEN ARCHIVS RAMSES' II.
}

Von Edgar B. Pusch und Stefan Jakob

\begin{abstract}
Abstrakt
In der Hauptstadt und Residenz der Ramessiden gelegen bei der Ortschaft Qantir im östlichen Nildelta Ägyptens - wurde das Randfragment eines diplomatischen Briefes vom hethitischen an den ägyptischen Königshof in tertiärer Lage oberhalb eines repräsentativen Gebäudes gefunden, das in die Zeit Ramses' II. datiert. Es gehört zu einer gröBeren Tafel, die möglicherweise die aktuellen zwischenstaatlichen Beziehungen zum Thema hat und in diesem Zusammenhang wohl den Pharao selbst in seiner Eigenschaft als „Herr der (beiden) Länder, d. h. Ober- und Unterägypten“ erwähnt.
\end{abstract}

\begin{abstract}
Within the Ramesside capital and residence situated in the Eastern Nile Delta, Egypt, the rim fragment of a cuneiform tablet was found, which belongs to the diplomatic correspondence between the Hittite and the Egyptian courts. Its position is to be defined as out of context, whilst it was found in a layer above a palace like structure dating to the time of Ramesses II. The text possibly reflects the current international relationship and mentions Ramesses himself as "Lord of the (two) Lands, i. e. Upper and Lower Egypt"
\end{abstract}

\section{ZUR EINSTIMMUNG}

Seit langen Jahren bestand die Hoffnung, ja der Wunsch, daß die Hildesheimer Grabungen in der Ramses-Stadt außer Ergebnissen zur Stadtstruktur und ihrer Entwicklung, zur Technologie der Ramessidenzeit und zu weiteren übergreifenden Fragen auch einen Beitrag zu den sicherlich vielfältigen Auslandsbeziehungen dieser Zeit und dieser Residenz liefern könne. Häufig war dies in der

1 Grabungsplatz Q VII-e/6, 7,25 m NS, 8,69 m WO, 4,765 $\mathrm{m}$ üNN, Schicht rel. a, Bauschicht rel. Ba.

${ }^{2}$ Nach grabungseigener Terminologie in Qantir-Süd gelegen, das laut der magnetischen Prospektion mehrere ungewöhnliche Gebäudegrundrisse enthält, die unter Begriff „Verwaltungsviertel“ zusammengefaßt wurden.; siehe dazu vorläufig Pusch 1999a.; 1999b, 20-24; Pusch/Becker/Fassbinder 1999a; 1999b. Lage
Geschichte des Projektes bereits der Fall: Die Anwesenheit ausländischer Götter wie Astarte, Reschef und Seth-Baal mag in diesem Zusammenhang wenig verwundern. Erinnert sei aber an die hethitischen Schildmodeln für Achterschilde und einen anatolischen Trapezschild, an die mykenische Keramik und an Waffenfunde im Kontext der Streitwagengarnison und ihrer Stallungen. Immer wieder wurde dann auch der - scherzhaft-ironische - Wunsch laut, daß wir doch bitte das Archiv Ramses' II. finden sollten: als Ergänzung zu dem Archiv von Hattusas/Boğazköy und der diplomatischen Korrespondenzen aus der Levante und als Parallele zur allseits bekannten Amarna-Korrespondenz. Daß nunmehr ausgerechnet im 25. Grabungsjahr des Projektes das Fragment einer Keilschrifttafel mit hethitischem Duktus gefunden wurde, hatte niemand ernsthaft erwartet.

\section{Der Befund}

Das Fragment lag in unmittelbarer Nachbarschaft zu einem Ofen, der mit großer Wahrscheinlichkeit der Herstellung von Rohglas diente (Abb. 1). ${ }^{1}$ Die betreffende Schicht, in die der Ofen eingebettet ist, war nur vom Ackerboden und einer hier recht dünnen Störungsschicht aus sandigem Lehm mit zahlreichen Kalksteinsplittern überdeckt. Die Einbettungsschicht des Ofens gehört zu den stratigraphisch spätesten Phänomenen des Grabungsplatzes Q VII, ${ }^{2}$ von dem auf Grund der magnetischen Prospektion ursprünglich angenommen worden war, daß er unter anderem möglicherweise einen hethitischen Tempel enthält, ein Gebäude, das inzwischen als rein ägyptische Architektur mit hoch offiziellem Charakter gesichert werden konnte. im Kataster der Region: Nord-West-Ecke $-\mathrm{x}=1433,477$, $y=1503,870$; Nord-Ost-Ecke $-\mathrm{x}=1458,147$, y=1498,362; Süd-West-Ecke - x=1421,516, y=1437,859; Süd-Ost-Ecke - 1443,006, y=1431,026. Zur Lage im Areal der RamsesStadt siehe besonders die Rekonstruktion der historischen Landschaft DORNER 1999 mit den beiden Faltplänen. 


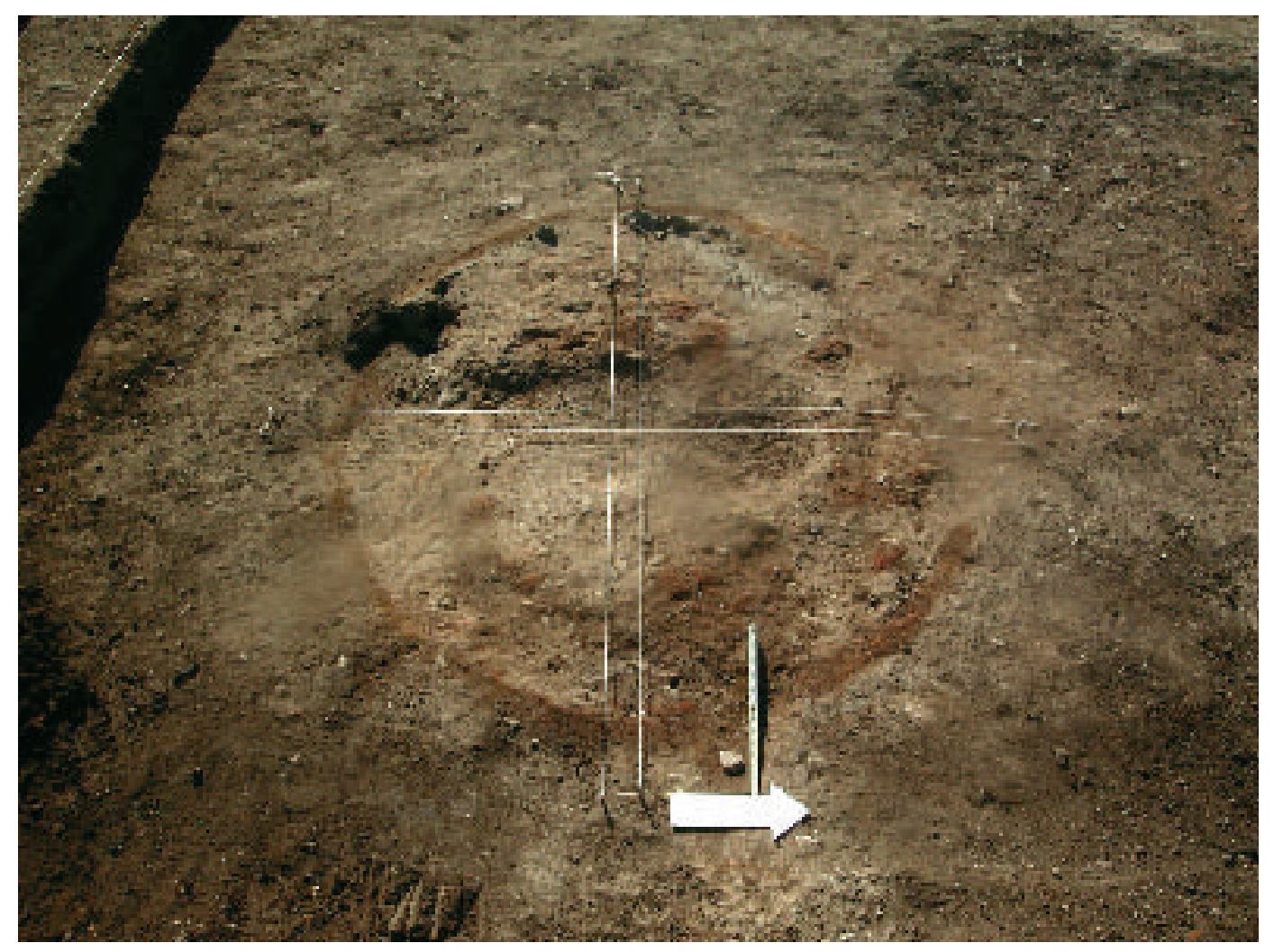

Abb. 1 Q VII-e/6, Schicht rel. a, Bauschicht rel. Ba - Ofen zur Herstellung von Rohglas (Ø 2,00 m) mit Fragment einer Keilschrifttafel FZ 2003/0260 am Ort der Auffindung. (Photo: E.B. Pusch)

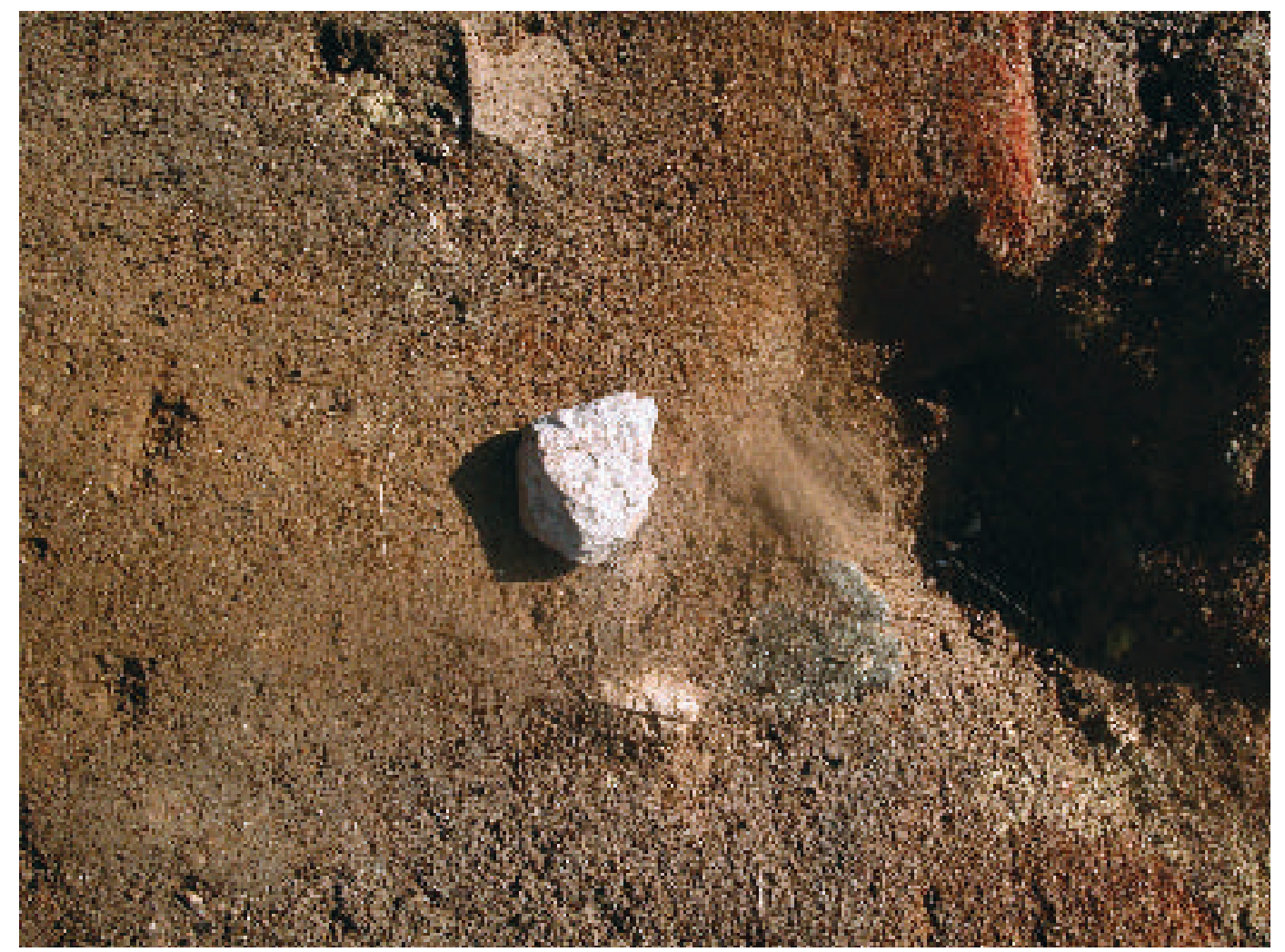

Abb. 2 Q VII-e/6, Schicht rel. a - Keilschrifttafel FZ 2003/260 am Ort der Auffindung, ehemals eingebettet in verziegelten Lehm neben einer Öffnung in der Ofenwand. (Photo: E.B. Pusch) 
Die Schicht, an deren Oberkante das Keilschrifttafelfragment lag und in die die Ofengrube eingreift, besteht aus einer mehrfach geschichteten Kalksteinschüttung mit Einschüben von stark humos-aschigen Zonen, die den ehemals potentiellen hethitischen Tempel überlagern bzw. überschneiden. Die gesamte Schichtgruppe ist unabweisbar nachzeitig zu der ansonsten innerhalb des Grabungsplatzes festgestellten Architektur. Architektonische Elemente, die mit der Schicht des Ofens verbunden werden könnten, sind bisher nicht vorhanden: Im Norden, Süden und Westen erstrecken sich ausgedehnte Störungen, die alle vorhandene Architektur überschneiden; unmittelbar im Osten befindet sich ein rezenter, gemauerter Bewässerungskanal, der zur Zeit die Grenze des angemieteten Grabungsplatzes bildet.

Das eigentliche Einbettungsmaterial der Keilschrifttafel ist ein in situ verziegelter Lehm, dessen Verziegelung durch eine vermutlich kreisförmige Öffnung - nur ihre Unterkante ist erhalten - in der Ofenwandung bewirkt wurde (Abb. 2). Dieser verziegelte Lehm erstreckt sich von einer Öffnung in der Ofenwand keilförmig ausgreifend nach Osten und umschloß das Fragment vollständig. Das weiter umgebende Sediment besteht aus einem braunen, homogenen Lehm, in den ungeregelt liegende Keramik, Holzkohle, Kalksteinsplitter und Kalksteinbrocken eingebettet sind. Eine Baugrube für den Ofen ist in Höhe des Auffindungsniveaus nicht vorhanden. Daß es sich bei der Auffindungshöhe um das Betriebsniveau des Ofens - bzw. nur ganz knapp darunter - handelt, dafür spricht auch die kleeblattförmige Ausformung des Ofenmundes mit seinem Gefälle zur Ofensohle und die in ihm sowie dem Ofen selbst enthaltenen Sedimente und Merkmale, zu denen ausgeprägte Ascheschichten, verschlackte Ofenwandungen und vor allem die Oxydationszonen der Ofenwandung selbst gehören. Sie verlaufen in Höhe der Unterkante der Lagerung der Keilschrifttafel und unmittelbar neben der Öffnung in der Ofenwand von innen nach außen von tiefrot - also stark verziegelt - über hellrot zu rotbraun - also leicht verbrannt.

Das Keilschrifttafelfragment lag mit der extrem erodierten Seite nach oben weisend flach in den verziegelten Lehm eingebettet. Die Randwölbung wies nach Südosten (s. Abb. 2). Als Fragment einer Keilschrifttafel wurde es erst nach dem Aufnehmen und einer vorläufigen Reinigung im Feld erkannt, da es in dem extrem feuchten und schlammigen Sediment einem ganz normalen, erodierten Gefäßfragment glich.
Bei diesem Gesamtbefund kann es sich nur um eine tertiäre Lagerung der Keilschrifttafel handeln, also eine Lagerung außerhalb des Nutzungskontextes und der Nutzungsschicht. Die Fragen, warum und wie das Fragment in diese Lagerung gelangte, warum es ausgerechnet neben einen Ofen zu Rohglasherstellung zu liegen kam, werden nicht zu klären sein. Die Suche nach weiteren Fragmenten in der unmittelbaren Umgebung - im Ofeninneren und seinem Umfeld - blieb bisher erfolglos. Das Tafelfragment ist also sowohl horizontal als auch vertikal verzogen worden; dafür, daß es zwischenzeitlich länger an einer Oberfläche gelegen hat und dort bewegt wurde, spricht die Form seiner Verwitterung. Dennoch besteht die Hoffnung, im Umfeld - also sowohl in der Tiefe als auch in der näheren Umgebung - weiteres zu finden. Eine Klärung in dieser Hinsicht war allerdings in der Kürze der zur Verfügung stehenden Zeit nicht möglich. Diese Arbeiten sollen fortgesetzt werden.

\section{Die Schichten Und ihre Datierung}

Da im unmittelbaren Umfeld zur Tafel bisher keinerlei Architektur erfaßt werden konnte, da sie in der obersten der ungestörten Schichten lag, kann ihre stratigraphische Einordnung und ihre aus Schichtbefunden zu erschließende Datierung nur über die früheren Schichten erfolgen. Inschriftliches Fundgut in primärer Lage zur Tafel ist ebensowenig vorhanden wie andere Kleinfunde, die aussagekräftig wären. Nach einer vorläufigen Durchsicht der Keramik des Grabungsplatzes aus den früheren Schichten, die von D. Aston bestätigt wurde, datieren alle Gefäße in primärer Lage in die 19. Dynastie. Das gilt auch für solche in sekundärer Lagerung. Diese Keramik gehört zu den verschiedenen Schichtpaketen des Gebäudes, das durch die Einbettungsschichten des Ofens überlagert wird. Innerhalb der Schicht, in die die Ofengrube einschneidet, wurden Gefäße beobachtet, die laut D. AsTON in die 20. Dynastie datieren. Zur Zeit ist noch nicht bekannt, ob sie in primärer oder sekundärer Lagerung aufgefunden wurden, in jedem Fall ist von einer Nachzeitigkeit zu dem Gebäude und seinen Schichten auszugehen.

Das Gebäude selbst weist eine reiche Stratigraphie auf. Es wurde auf mächtigen Fundamenten errichtet, die es mittels einer Plattform über das umgebende Siedlungsniveau heraushoben, sodaß im angetroffenen Erhaltungszustand Fußböden nur noch in geringer Ausdehnung erhalten waren. Sie umschließen die oben angesprochenen Gefäße.

An das Gebäude angebaut fanden sich Außen- 
räume, die durch einen einheitlichen Kalkverputz die Außenräume auf dem niederen Siedlungsniveau mit dem Gebäude auf der erhöhten Plattform zusammenbinden. Ab einem noch zu ermittelnden Zeitpunkt wurden also der Hauptbau und die Außenräume zeitgleich genutzt. In diesem Zusammenhang ist zweierlei von Bedeutung.

In einem der Außenräume fand sich bereits in der Kampagne 2002 ein Türsturz Ramses' II. ${ }^{3}$ in sekundärer Lage: Er war von Ost nach West in den Raum verstürzt und lag flach auf dem gekalkten Ziegelpflaster des Raumes auf. Seine ehemalige Position läßt sich über das Magnetbild rekonstruieren. Die betreffenden Räumlichkeiten und alle mit ihnen verbundene Architektur können also problemlos in die Zeit Ramses' II. gestellt werden.

Den zweiten Datierungsansatz bietet ein Fußboden des Vorhofes des Gebäudes. Er erstreckt sich in dessen Norden über eine Fläche von mindestens 35 $\mathrm{m}$ auf $15 \mathrm{~m}$. Während auf ihm keinerlei Fundgut angetroffen wurde, liefert sein komplexer Aufbau reiches Material. Vollkommen eben angelegt, besteht er aus einem mit einer Kalkschlämme getränkten Sand der auf einer dicken, bis zu $25 \mathrm{~cm}$ umfassenden, verdichteten Kalksteinrollierung aufliegt, die ihrerseits auf einem Sandbett ausgebreitet wurde. Diese Kalksteinrollierung besteht aus zerschlagenen Architekturelementen mit Inschriften und Darstellungen in erhabenem und versenktem Relief mit polychromer Fassung von herausragender Qualität. Neben Teilen von Türeinfassungen und einer Deckenverkleidung scheint die Masse der Fragmente zu einer ausgedehnten Wandverkleidung zu gehören. Auf ihnen konnte vielfach der Name Ramses' II. identifiziert werden.

Während das Verhältnis der beiden hier angesprochenen Schichten zueinander noch nicht abschließend geklärt ist, kann dennoch festgehalten werden, daß das Gebäude, dem die Schicht mit dem Glasofen aufliegt, zumindest in Teilen in die Zeit Ramses' II. datiert, wobei für den jüngsten seiner Vorhof-Fußböden ein Terminus ante quem non gewonnen wurde.

Zusammenfassend läßt sich daraus ableiten, daß die Einbettungsschicht der Keilschrifttafel in die Zeit Ramses' II. oder kurz danach datiert werden muß. Letzteres gilt, wenn sich durch weitere Untersuchungen herausstellt, daß die „Ofenschicht" tatsächlich in der 20. Dynastie angesetzt werden muß. Sowohl weitere Grabungen als auch eine detaillierte Untersuchung der Keramik bleiben hier abzuwarten. In jedem Fall aber sind alle Schichten als „Ramessidisch" anzusprechen und damit in einem Zeitraum zwischen ca. 1250 bis ca. 1150 v. Chr. anzusiedeln. ${ }^{4}$

\section{DAS ObJekT}

Das Keilschrifttafelfragment ${ }^{5}$ ist auf beiden Seiten stark erodiert (Abb. 3). Eine Entscheidung über Vorder- und Rückseite ist daher nicht möglich. Es handelt sich um ein Randstück, das rechtslateral die übliche Rundung, linkslateral sowie oben und unten Brüche aufweist. ${ }^{6}$ Alle Brüche sind alt. Seine Maße betragen: Höhe: $\mathrm{x}+4,96+\mathrm{y} \mathrm{cm}$, Breite: $\mathrm{x}+4,95 \mathrm{~cm}$, Dicke: 2,0 $+\mathrm{x} \mathrm{cm}$ (maximal).

Der Werkstoff besteht aus einem fein levigierten, alluvialen Ton ohne makroskopisch erkennbare organische oder anorganische Magerung; unter dem Fadenzähler ${ }^{7}$ sichtbarer Glimmer dürfte dem Einlagerungssediment entstammen. In den Brüchen der Ober- und Unterseite sowie auf der Rückseite wird eine deutliche Schichtung sichtbar, die besonders im oberflächennahen Bereich eine dünne Zonenbildung aufweist. Längs dieser Schichtung ist der linke Teil der „Vorderseite“ abgeschiefert. Alle Kanten sind verrundet. Das offensichtlich gebrannte Fragment weist an der erhaltenen Oberfläche eine dunkelrote $\left(2.5\right.$ YR 5/4), ${ }^{8}$ bei abgeplatzter Oberfläche eine braunrote (5 Y 6/3) Färbung auf. Im Kern ist es dunkelgrau (2.5 YR 4/0 bis 5 YR 5/1). Daß beide Oberflächenbereiche einschließlich der Rundung „rot“, der Kern aber weitgehend „schwarz“ gefärbt sind, spricht dafür, daß es trotz der Lagerung neben der Ofenöffnung nicht sekundär, sondern primär, also intentionell, gebrannt wurde. Bei einem sekundären Brand durch die aus dem Ofenloch austretende Hitze hätten die zum Ofen hin orientierten Brüche stärker gebrannt sein müssen, als die übrigen Partien der Tafel.

${ }^{3}$ Fundzettelnummer (FZN): 2002/0334.

4 Vgl. dazu BeCKerath, 1971.

5 FZN: 2003/0260, Funddatum: 30. August 2003, Befundtext: „in verziegeltem Lehm neben Ofen nach Abtrag der K(alkstein)Splitt(schicht) rel. a“, Inventarnummer: IN 2801 (Magazin des Projektes Ramses-Stadt).

\footnotetext{
${ }^{6}$ Orientierung bezogen auf die besser erhaltene Seite.

7 Bei zehnfacher Vergrößerung.

8 Farben nach Munsell Soil Color Chart, 1975; beurteilt in diffusem hellem Sonnenlicht.
} 
a)
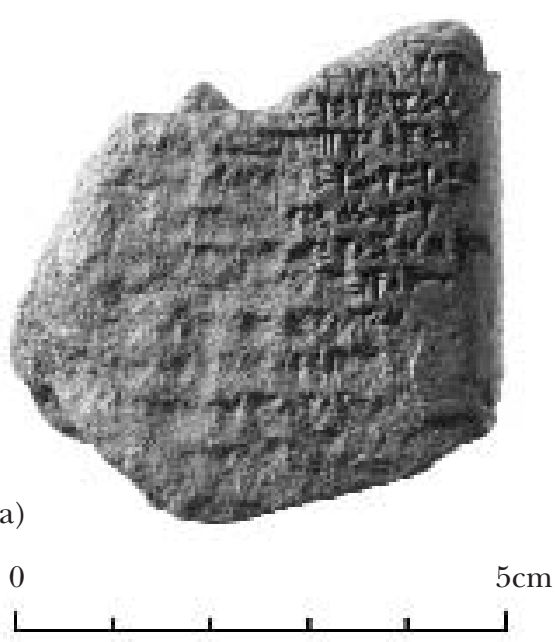

c)

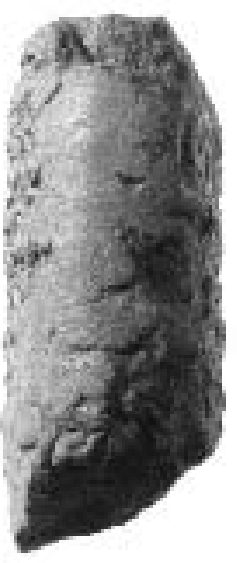

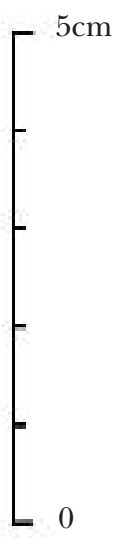

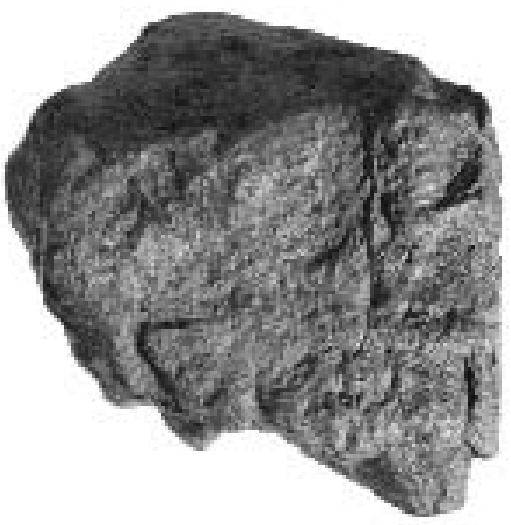

b)
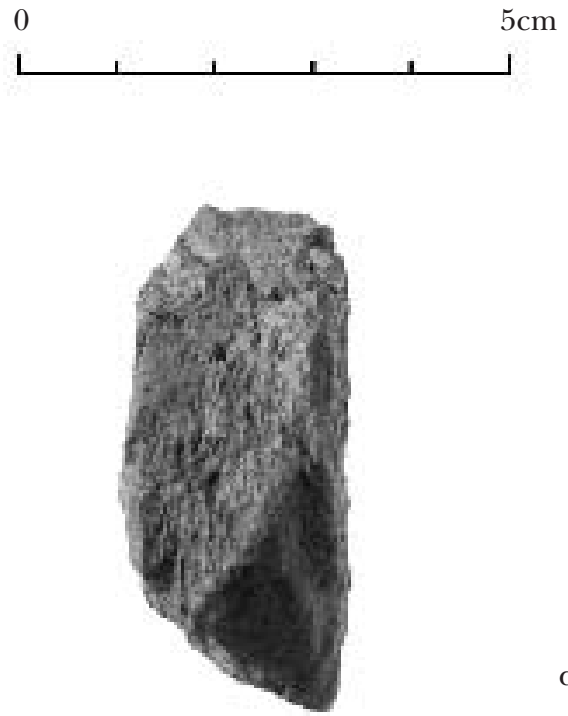

d)

Abb. 3 Keilschrifttafel FZN 2003/0260: a) Vorderseite; b) Rückseite; c) rechtslateraler Rand;

d) linkslateraler Bruch; Maßstab 1:1 (Photos: K. PARLOW)

Erhalten sind auf der „Rückseite“, die bei der Auffindung sichtbar war, nur die Reste einiger weniger Zeichen, verteilt auf mehrere Zeilen und nur in den höchst erhaltenen Abschnitten (Abb. 3, 4). Ansonsten besteht sie aus einer wahren Kraterlandschaft, in der die Schichtung des Werkstoffes mit den oben angegebenen Farbabstufungen bis hin zum schwarzen Kern sichtbar wird.

Die „Vorderseite“ bietet die Enden von $\mathrm{x}+11+$ y Zeilen, von denen die Enden von wiederum 9 Zeilen scharf erhalten sind (Abb. 5). Die Oberfläche des linken Teils der Vorderseite ist so abgeplatzt, daß nur mehr die tiefsten Eindrücke der Keilschriftzeichen vorhanden sind. Zum linkslate- ralen Bruch hin verschwinden auch die tiefsten Zeichenreste.

Das Objekt wurde unter dem Mikroskop mit einem Skalpell mechanisch gereinigt. Die Oberflächenpatina, die insbesondere in den geschichteten Zonen der Brüche vorhanden ist, wurde belassen. Abschließend wurde das Tafelfragment mit einer zweiprozentigen Acrylharzlösung überzogen, um gefahrloses Handhaben zu ermöglichen. ${ }^{9}$

\section{Der TeXT UND SEIN INHALT}

Das Fragment 2003/0260 ist der rechte Rand des oberen mittleren Teils einer Tontafel von ursprünglich wohl mindestens $10 \mathrm{~cm}$ Höhe. Bei voller Aus-

\footnotetext{
${ }^{9}$ Carla Leupold, Hochschule für Angewandte Wissenschaft und Kunst, Hildesheim.
} 
nutzung des dann zur Verfügung stehenden Raumes läßt sich auf ungefähr 60 oder mehr Zeilen mit jeweils 25-30 Zeichen schließen. ${ }^{10}$ Die Sprache ist Akkadisch, die lingua franca des Vorderen Orients im zweiten vorchristlichen Jahrtausend. Wir lesen auf dem Fragment zunächst das Folgende:

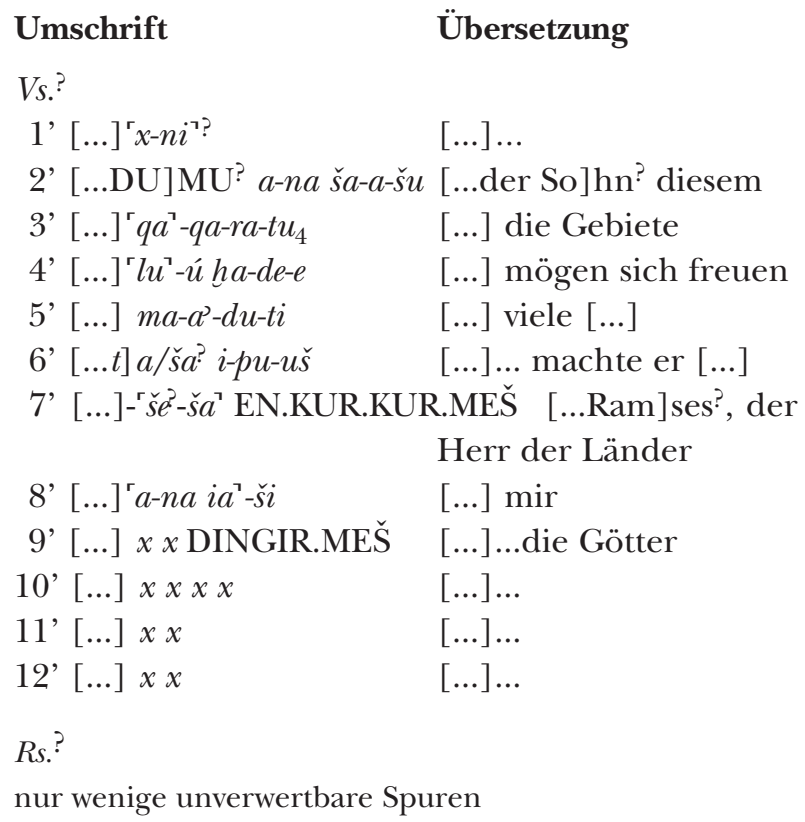

Aus Zeile 8' geht klar hervor, daß es sich um einen Brief handeln muß. Auch wenn weder Absender noch Adressat bekannt sind, deuten Indizien in eine bestimmte Richtung. Der Fundzusammenhang verweist auf die Regierungszeit Ramses' II. (s. o.). Zudem darf in Zeile 7', wie noch zu zeigen sein wird, der Name Ramses in seiner keilschriftlichen Umsetzung Riamašeša (geschrieben I Ri-a-ma-še-ša) mit hoher Wahrscheinlichkeit ergänzt werden.

Es liegt daher nahe, den Blick auf die Korrespondenz dieses Pharaos zu richten. In der Folge der Schlacht von Qadeš zwischen Ramses und dem Hethiterkönig Muwatalli hatten sich nach jahrelangen, für beide Seiten aufreibenden Auseinandersetzungen die diplomatischen Kontakte intensiviert und schließlich in Ramses' 21. Regierungsjahr in die Vereinbarung des ersten internationalen Friedensvertrags der Geschichte gemündet. ${ }^{11}$ Teile der Korrespondenz zwischen Ägypten und Hatti wurden in Hattuša (modern Boğazköy), der Hauptstadt der Hethiter, geborgen. Die Schreiben beziehen sich u. a. auf das Verhältnis beider Länder vor und nach
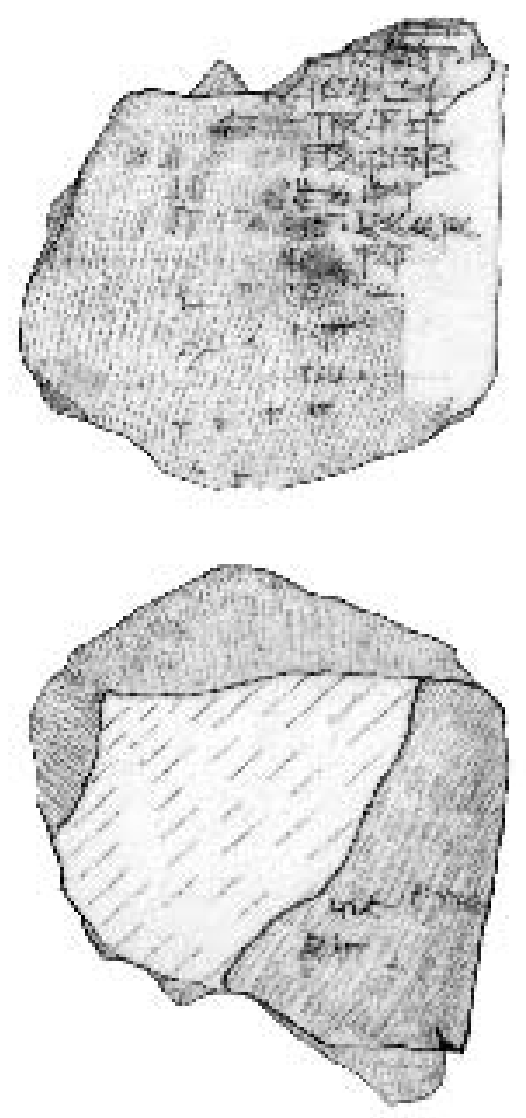

Abb. 4 Keilschrifttafel FZN 2003/0260, Faksimile Maßstab 1:1 (Zeichnung: S. ЈАКОВ)

dem Friedensschluß, erwähnen Geschenksendungen oder sollen zur Klärung der Modalitäten beitragen, unter welchen Umständen und mit welcher Mitgift eine hethitische Prinzessin als Braut an den Hof Ramses II. zu geleiten sei.

Ob unser Fragment zur ägyptisch-hethitischen Korrespondenz gezählt werden kann, ist aufgrund des schlechten Erhaltungszustands nach inhaltlichen Kriterien kaum endgültig zu entscheiden. Daher müssen zunächst Zeichenformen und Syllabar zur Argumentation mit herangezogen werden. Von besonderem Interesse hierbei sind natürlich die Briefe der hethitischen Seite, ${ }^{12}$ seien es Entwürfe oder Kopien, da man annehmen darf, daß diese aus derselben Kanzlei stammen wie ein tatsächlich nach Ägypten versandtes Schreiben.

Ausgesprochene „Leitzeichen“, die eine sichere paläographische Einordnung erlaubten, fehlen leider. Allerdings finden sich für die von der Stan-

\footnotetext{
10 cf. Edel 1994, Tafel XLIVf.

11 DERS. 1997.
}

12 s. Hagenbuchner 1988. 


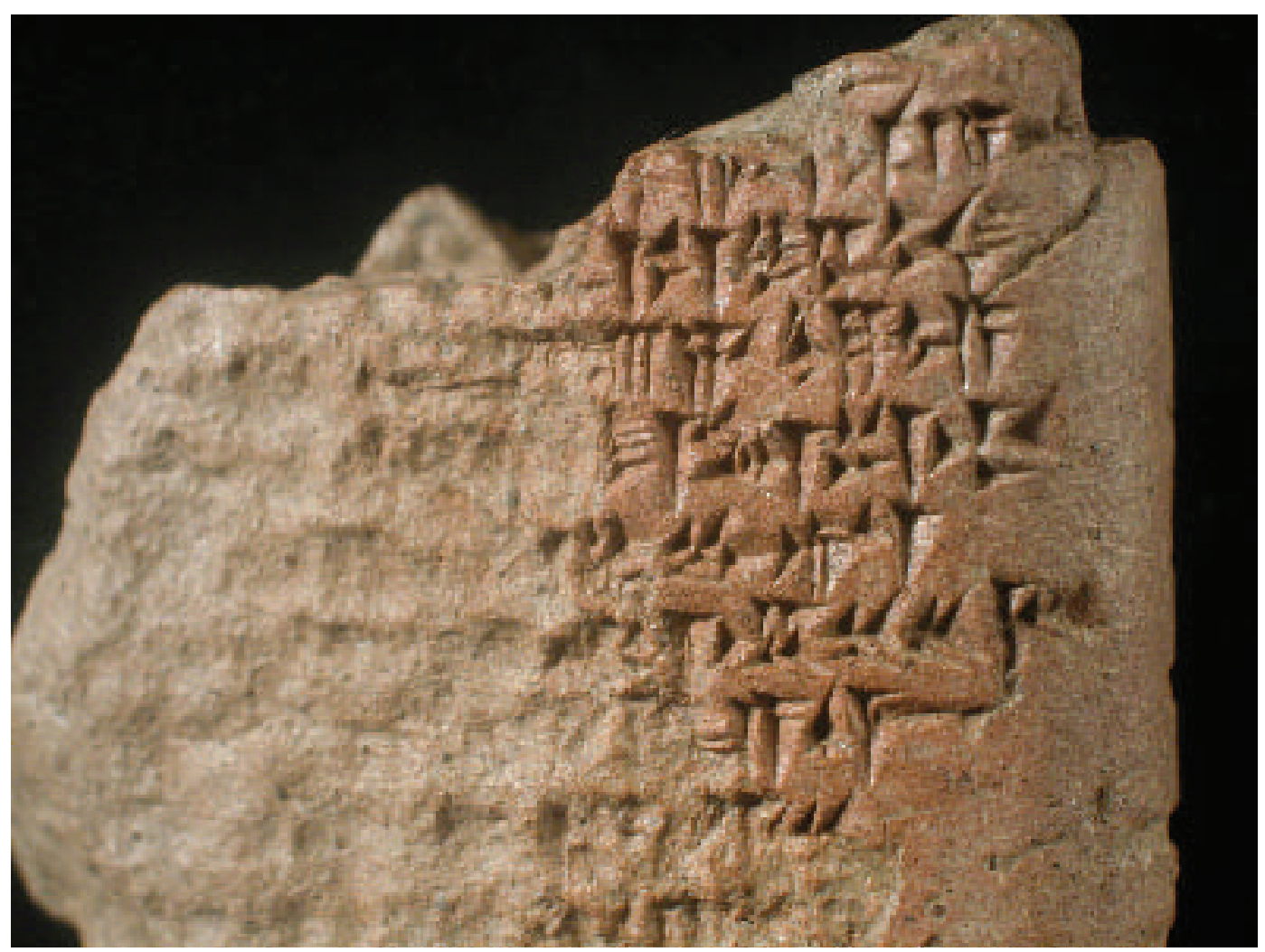

Abb. 5 Keilschrifttafel FZN 2003/0260, Detail der Vorderseite (Photo: K. PARLOW)

dardform abweichenden Zeichen Parallelen in publizierten Briefen, sei es für $u \check{s},{ }^{13} u^{14}$ oder $r a{ }^{15}$ Die hypertrophe Schreibung des „ma“ gelesenen Zeichens in Zeile 5' ist sonst meines Wissens in den Briefentwürfen aus Hattuša nicht belegt. Das spricht aber weder gegen die vorgeschlagene Lesung noch gegen eine hethitische Provinienz des Fragments, zumal innerhalb der hethitischen Kanzlei einzelne Zeichen, bisweilen sogar innerhalb eines einzigen Briefes, mehr oder weniger stark abweichende Varianten bilden.

Hinzuweisen bleibt auf die Schreibung der

13 KUB III 40 (zwischen Vasall und Großkönig oder zwischen zwei Großkönigen; s. ibd., No. 341).

14 KBo XXVIII 68 Rs. 10' (ibd., No. 319)

15 KUB III 46 Vs. 5 (Hattušili ? an Ramses ?; s. EDEL 1994, 41)

$16 i b d$., E 20 Vs. 17' (KUB III 24+KUB III 59)

17 s. RÜster/NeU 1989, No. 332.

18 v. Soden/Röllig 1991, No. 233.

19 Bestimmte Zeichen wie etwa „ti“ (cf. Z. 5') wären von assyrischen oder babylonischen Schreibern an den jeweiligen Königshöfen so nicht geschrieben worden. Auch Hanigalbat, ein weiterer potentieller Briefpartner des Pharao scheidet aus, wie KBo XXVIII 66 (=HAGEN-
Silbe „ $a$ ”. Diese wird nach hethitischem Usus in der Regel, etwa in einem Brief der hethitischen Königin Puduhepa an Ramses, ${ }^{16}$ mit dem Zeichen $a h^{17}$ ausgedrückt, während die in 2003/206 gebotene Form die in Mesopotamien übliche ist. ${ }^{18}$ Es wäre allerdings verfehlt daraus schließen zu wollen, der Brief sei zwangsläufig von dort gekommen, ${ }^{19}$ zumal die „mesopotamische“ Form auch in einem Schreiben Hattušilis III. an den babylonischen König Kadašman-Enlil II. verwendet wird. ${ }^{20}$ Das macht eine Urheberschaft des hethitischen Königs doch wahrscheinlich. ${ }^{21}$
BUCHNER 1988, No. 211) erweist, wo Z. 5 das Zeichen ah zum Ausdruck des Alef verwendet wird.

${ }^{20}$ KBo I 10:60 (= ibd., No. 204).

${ }^{21}$ Hier wäre zu fragen, ob fallweise seitens des hethitischen Königs jeweils bestimmte, in Sprache und Ausdruck des Adressaten speziell geschulte Schreiber beauftragt wurden. Man vergleiche hierzu etwa den Brief KBo I 14, zu dem A. Hagebuchner, op. cit., 267 schreibt: „Auf Grund der...zahlreichen Assyriasmen...ist das Schreiben von einem hethitischen König an einen assyrischen König adressiert worden“. 
Es gibt - theoretisch - noch eine weitere Möglichkeit: Die Schreibung des Alef als $a^{2}$ findet sich auch in Briefen des Pharao an Hattušili III. oder Tuthaliya IV.22 Diese Tafeln gehören zu den sogenannten insibja-Briefen Ramses' II., die zwischen dessen 42. und 56. Jahr anzusetzen sind. Gegen die Annahme, es handele sich bei unserem Fragment um einen ägyptischen Briefentwurf spricht allerdings Z. 7': Das Sumerogramm EN.KUR.KUR.MEŠ ist akkadisch bēl mätāti zu lesen. Das ist zwar als ungefähre ${ }^{23}$ Entsprechung zu ägyptisch $n b t 3 w j$ „Herr der beiden Länder" tatsächlich Teil der Titulatur des Pharao und paßte hervorragend zu der eingangs vorgeschlagenen Ergänzung [Ri-a-ma] $-\check{s}^{2} e^{3}-\check{s}^{2} a^{3}$. Problematisch scheint allerdings, daß die ägyptischen Schreiber in allen bekannten Fällen ni-ib ta-a-ui schreiben, also das Wort ihrer Sprache stets lautschriftlich in die Keilschrift überführen. ${ }^{24}$ Der Titel bèl mātāt $i$ ist im Übrigen im eigentlichen akkadischen Sprachraum selten. In Texten des 2. Jahrtausends findet er sich nur als Epitethon der Götter Enlil ${ }^{25}$ und Aššur. ${ }^{26}$ Als Königstitel ist er erst in der Endphase des neuassyrischen Reiches nachweisbar. ${ }^{27}$

Die vorgestellten Indizien verweisen also letztlich für 2003/0260 mit hoher Wahrscheinlichkeit auf den hethitischen König als Absender und Pharao Ramses II. als Adressaten. Was der eine dem andern mitzuteilen hatte, können wir nicht mit Bestimmheit sagen, es seien aber zum Abschluß, einige, wenngleich zugegebenermaßen sehr spekulative Gedanken zum Inhalt des Schreibens erlaubt:

Die Verbform' $l u^{\prime}-u$ ha-de-e Z. 4' ist durchaus bemerkenswert. Wenn die Partikel "lī" richtig ergänzt und daher nicht vom Genitiv phad $\hat{u}$ „Freund“ auszugehen ist, handelt es sich um einen Stativ f. pl. mit Kontraktion (< hadia $)$, wie sie sonst für die Sprache des altbabylonischen Mâri bekannt ist, nicht aber für die hethitische Korrepondenz des späten 2. Jahrtausends. Zugehöriges Subjekt wäre dann möglicherweise qaqqarātu in Z. 3'.

${ }^{22}$ KUB III 28 Vs. 4 (= EDEL 1994, No. 69); KUB III 44 Vs. 21 (=EDEL 1994, No. 74).

23 KUR.KUR.MEŠ muß nicht unbedingt Ober- und Unterägypten bezeichnen; s. hierzu etwa $i b d$., 110 (Fremdländer an der Seite der Hethiter bei der Qadě́Schlacht).

24 s. ibd., 168-177; zur Übertragung von hieroglyphischen Titeln in Keilschrift s. ibd. 36.

${ }_{25}$ altbabylonisch bēlum...ša KUR.KUR.RA-tim (RA 39,6,4); mittelassyrisch EN KUR.KUR in der Einleitung einer
Die ersten erhaltenen Zeilen könnten, wenn die Spuren richtig gedeutet wurden, somit auf einen Sohn anspielen, der zu einem anderen, etwa seinem Vater, in einer bestimmten Beziehung steht. Wenn sich darauf "Gebiete“ (= Distrikte eines Verwaltungsgebietes ?) freuen sollen, mag man darin im äußersten Fall einen Thronwechsel vermuten.

In diesem Sinne wäre Z. 5' in Anlehnung an $\S 10$ des ägyptisch-hethitischen Friedensvertrages (s. Anm. 10) vor $m a-a^{3}-d u-t i$ etwa MU.MEŠ "Jahre“ zu ergänzen und damit das Inkraftreten jener Vereinbarung zu postulieren, wonach Ramses dem Sohn des Hattušili III. bei seiner Thronbesteigung gegen eventuelle Widersacher im eigenen Land beistehen sollte. Es ergäbe sich folgender Sinn: „Viele Jahre hat er (Hattušili) als König geherrscht, nun aber...". Dabei müsste angesichts der vielfältigen Verwendung von epēsu "machen“ als Verb in der ägyptisch-hethitischen Korrespondenz in stehenden Redewendungen mit „Bruderschaft, Freundschaft, Vorschlägen, Plänen, Eiden“, aber auch „Verfehlungen“ oder einer anzufertigenden „Silbertafel“" offen bleiben, ob ipuš in Z. 6' noch in denselben Zusammenhang gehört oder bereits den nächsten Gedankengang abschließt. Die Verwendung des Präteritums mag jedenfalls darauf hindeuten, daß einer in der entfernteren Vergangenheit erfolgten oder begonnenen Handlung gedacht wird. ${ }^{28}$

Danach wird Ramses unmittelbar angesprochen, ehe der Absender noch einmal auf sich verweist, etwa dahingehend, daß die Zeit für den Pharao gekommen ist, sich seines vertraglich verbrieften Versprechens zu erinnern. Hierzu würde auch die Erwähnung der Götter in der folgenden Zeile passen.

\section{Der Grabungskontext}

Bereits eingangs wurde angeführt, daß das Tafelfragment keineswegs isoliert im Gesamtkontext der Grabung steht. Schon der Grabungsplatz Q I hatte

Prisma-Inschrift des assyrischen Königs Tiglatpilesar I. (A.O.87.1 I 4; GRAYSON 1991, 12).

${ }^{26}$ Sg. 8, 116, 314 (Thureau-Dangin o.J.).

27 SAA X 196 Rs. 4: EN KUR.KUR. Vielleicht ist auch von dem Gelehrten, der sich hier brieflich an den König wendet, ein Bezug zur göttlichen Sphäre intendiert, indem er die Titulatur des obersten Gottes Ǎšsur, der im Übrigen in der assyrischen Ideologie mit Enlil geglichen wird, auf den König überträgt.

28 cf. v. SODEN 1969, § 79. 
hethitisches Kulturgut enthalten: Acht Treibmodeln für die Herstellung von metallenen Schildbeschlägen, verteilt auf sechs Kalksteinplatten wurden dort gesichert, von denen eine sich nebst dazu gehörendem Werkzeug in primärer Lage befand. Die Identifikation eines dieser Plattenmotive als hethitischer Achterschild ist über die Darstellungen der Qadesch-Schlacht und einen Orthostaten des 9. Jahrhunderts v.Chr. aus dem späthethitischen Sencirli möglich. Das andere Motiv mag einstweilen als anatolischer Trapezschild angesprochen werden. ${ }^{29}$ Ferner kann in diesem Zusammenhang ein Stelenfragment genannt werden, das einen „(Magazin)Verwalter" anatolischer Abstammung namens Arzawija, „Der Arzawäer“30 und seinen Kollegen Sethi, „Der zu Seth (oder zu Sethos?) gehörige“ nennt, wobei letzteres durchaus auch als Baali, „Der zu Baal gehörige“ gelesen werden kann. ${ }^{31}$ Neben diesen unmittelbaren anatolischen Bezügen steht ein reiches Fundgut aus dem mykenischen Raum der Späten Bronzezeit, wobei nicht nur geschlossene GefäBe als Container kostbarer Öle, sondern auch offene Formen als luxuriöses Tafelgeschirr vorhanden sind. ${ }^{32}$ Nicht unerwähnt bleiben darf die Schuppe von einem mykenischen Eberzahnhelm ${ }^{33}$ und ein bisher noch rätselhafter Scherben mit vermutlicher Rebusritzung, die der Kommunikation zwischen einem Ägypter und einem Nicht-Ägypter gedient haben könnte. ${ }^{34}$ So, wie das Fragment der hier vorgestellten Keilschrifttafel nur der Zipfel des diplomatischen Archives der Ramessidenzeit innerhalb der Residenz darstellt, stellt das übrige Fundgut nur die Spitze des Eisberges der intensiven Auslandsbeziehungen in der Ramessidenzeit dar. Diese erstrecken sich keineswegs nur auf die materiellen

29 Siehe dazu vorläufig Pusch 1993, 138ff., Abb. 135-138; englische Version Pusch 1996, 140ff., 135-138.

30 FZN 1982/0268: z3w Irtw (in Gruppenschreibung).

31 deographisch mit dem Seth-Tier geschrieben, wie auch der klare Seth-Baal in der Vierhundertjahr-Stele, siehe dazu z.B. Cornelius, 1994, BR5 und passim.

32 Mommsen et al. 1996 und Mountjoy/Mommsen 2001.

33 Pusch 1999a, 138 und Abb. 134.

${ }^{34}$ Pusch 1999b, 29 (4.4.6 Ostrakon mit Linear-B-Zeichen?) mit Abb. 3; dort nur angedeutet könnte die Zeichenkombination als „Stall für Pferde“ zu deuten sein, wobei das Hauptzeichen den Stall in seinem Grundriß, der Equidenkopf die Spezifikation darstellt.

35 EDEL 1976, 105. Auch die Pflege ausländischer Musik ist in diesen Zusammenhang zu stellen, stellen doch
Hinterlassenschaften, vielmehr ist zu fragen, ob und in welchem Umfang solche Grabungsinhalte wie „Glasproduktion“, „Streitwagenbau“, „Pferdetraining“, „Waffentechnologie“ und anderes einen stetigen und engen Technologie- und Wissenstransfer zwischen den Herrschaftsgebieten und Kulturkreisen des Vorderen Orients dokumentieren. Beispielhaft mag hier die Anforderung von Spezialisten aus Hupišna durch Ramses II. ${ }^{35}$ genannt sein.

\section{ZUSAMMENFASSUNG}

Jenseits aller anderen Überlegungen zur Identifikation der Region Qantir / Tell el-Dabca als dem Gebiet von Piramesse und Auaris, die hier nicht weiter verfolgt werden müssen, liefert das Tafelfragment mit seinem Inhalt einen weiteren Mosaikstein zur positiven Argumentation. Als erster Tafelfund in Ägypten - noch dazu unter kontrollierten stratigraphischen Bedingungen - seit der Sicherung der Amarna-Korrespondenz, deren überwiegender Teil 1887 bei Sebbachgewinnung auftrat, ${ }^{36}$ ist er gleichzeitig der erste Fund aus dem sicherlich umfangreichen diplomatischen Archiv Ramses' II. ${ }^{37}$ bzw. der Ramessidenzeit überhaupt. Wie andere Funde außerhalb Ägyptens und die Amarna-Briefe zeigen, ist insgesamt damit zu rechnen, daß das Archiv einst nicht nur eine intensive Korrespondenz mit dem hethitischen Königshaus, sondern mit allen führenden Häusern der Levante und des Zweistromlandes umfaßte. Auch dürfte es kaum auf die Zeit Ramses' II. allein beschränkt gewesen sein, wissen wir doch um die engen Kontakte noch Ramses III. mit dieser Gesamtregion. Mag der Erhaltungsgrad der sicherlich einst großen Tafel auch gering und bedauerlich sein, so zeigt einerseits ihr Auftreten überhaupt, mehrere Talatatblöcke hethitische Musikanten mit der großen Standharfe dar; s. dazu ManNiche 1975 (z.Zt. auf der Grabung leider nicht zugänglich).

36 Helck 1975, 173 mit weiterer Literatur.

37 Siehe dazu EDEL 1980, der lakonisch formuliert: „Von der ägyptisch-hethitischen Korrespondenz, die unter Ramses II. in babylonischer Sprache und Keilschrift mit dem hethitischen Königshaus geführt wurde, hat sich in Äg. selbst nichts gefunden, da die Hauptstadt Ramses' II. im Delta noch nicht ausgegraben worden ist." 
wozu intensive Feldforschung auch in einem so riesigen Areal wie dem der Ramses-Stadt in der Lage ist, andererseits bietet ihr Inhalt möglicherweise durchaus neue historische Nuancen, obzwar sich deren Relevanz an weiteren Funden erst noch erweisen muß. Auf jeden Fall regt das Fragment eine hoffentlich lebhafte und fruchtbare Diskussion an. Daß Grund zur Hoffnung besteht, weitere Teile des ramessidischen Archives zu sichern, darf erstmals ernsthaft in Erwägung gezogen werden. Daß das Projekt Ramses-Stadt unter der Gesamtleitung von ARNe EgGEBRECHT sich mit Hilfe der Deutschen Forschungsgemeinschaft (DFG), Bonn, darum intensiv bemühen wird -war sie es doch, die das Projekt über fünfundzwanzig Jahre großzügig finanzierte und förderte - davon dürfen wir alle ausgehen.

\section{Danksagung}

In einer außergewöhnlichen Situation bedarf es außergewöhnlicher Hilfe: So danken wir herzlich
S.E. Dr. Zahi Hawas, Chairman des Supreme Council of Antiquities, Ägypten, für sein Unterstützung bei der Beschaffung der Aufenthaltserlaubnis auf der Grabung, Prof. Dr. W. Röllig, der uns unter groBem Einsatz zusammenführte, Prof. Dr. GÜNTER DREYER, Deutsches Archäologisches Institut, Kairo, und Prof. Dr. MANFred BietaK, Österreichisches Archäologisches Institut, Kairo, für die Möglichkeit zur Nutzung ihrer Bibliotheksbestände auch vor Ort in Qantir.

\section{Persönliche Danksagung}

Danken möchte ich posthum als Finder der Tafel meinem Lehrer Elmar Edel, hatte ich doch während meiner Studienzeit das große Vergnügen, die Entstehung der Publikation der Keilschriftkorrespondenz zwischen den beiden Königshäusern in Vorlesungen und Übungen zu erleben.

Edgar B. Pusch 


\section{Bibliopgraphie}

BECKERATH, J. V.

1971 Abriss der Geschichte des Alten Ägypten, München, Wien.

CORNELIUS, I.

1994 The Iconography of the Canaanite Gods Reshef and $B a^{c} a l$, Fribourg, Göttingen.

DORNER, J.

1999 Die Topographie von Piramesse, $\ddot{A} \mathcal{E} L$ 9, 77-83.

EDEL, E.

1976 Ägyptische Ärzte und Ägyptische Medizin am Hethitischen Königshof, Rhein.-Westfälische Akademie der Wissenschaften. Vorträge G 205

1980 Königsbriefe, äg. aus Boğazköi, LÄ 3, 482-485, Wiesbaden.

1994 Die ägyptisch-hethitische Korrespondenz (ÄHK), Opladen

1997 Der Vertrag zwischen Ramses II. von Ägypten und Hattusili III. von Hatti, Berlin

GraYSON, A. K.

1991 Assyrian Rulers of the Early First Millennium I, Toronto

Hagenbuchner, A.

1988 Die Korrespondenz der Hethiter, Heidelberg

HeLCK, W.

1975 Amarna-Briefe, $L \ddot{A}$ 1, 173-174, Wiesbaden.

MANNICHE, L.

1975 Ancient Egyptian Musical Instruments, MÄS 34, Berlin Mommsen, H./Beier, T./Hein, A./Podzuweit, C./ Pusch, E. B./Eggebrecht, A.

1996 Neutron Activation Analysis of Mycenaean Sherds from the Town of Ramesses II near Qantir and Greek-Egyptian Trade Relations, Archaeometry 94 The Proccedings of the $29^{\text {th }}$ International Symposium on Archaeometry, Ankara 9.-14. May 1994, Ankara, 169-178.
Mountjoy, P. A./Mommsen, H.

2001 Mycenaean Pottery from Qantir-Piramesse, Egypt, The Annual of the British School at Athens 96, 123-155.

Munsell Soil Color Chart, Edition 1975, Baltimore, Maryland.

Pusch, E. B.

1993 ,Piramesse-geliebt-von-Amun, Hauptquartier Deiner Streitwagentruppen', Ägypter und Hethiter in der Delta-Residenz der Ramessiden, in: A. EGGEBRECHT (Hg.), Pelizaeus-Museum Hildesheim, Die Ägyptische Sammlung, 126-143.

1996 'Pi-Ramesses-Beloved-of-Amun, Headquarters of thy Chariotry', Egyptians and Hittites in the Delta Residence of the Ramessides, in: A. EgGEBRECHT (ed.), Pelizaeus-Museum Hildesheim, The Egyptian Collection, 126-144.

1999a Towards a map of Piramesse, Egyptian Archaeology $14,13-15$.

1999b Vorbericht über die Abschlußkampagne am Grabungsplatz Q IV, ÄEL 9, 17-37.

Pusch, E.B./Becker, H./Fassbinder, J.

1999a Palast - Tempel - Auswärtiges Amt?, ÄELL 9, 135-153.

1999b Wohnen und Leben oder: Weitere Schritte zu einem Stadtplan der Ramses-Stadt, $\ddot{A} \mathcal{E} L$ 9, $155-170$.

RÜster, C./NeU, E.

1989 Hethitisches Zeichenlexikon,Wiesbaden

v. SODEN, W.

1969 Grundriss der akkadischen Grammatik, Rom

v. Soden, W./Röllig, W.

1991 Das akkadische Syllabar, Rom

Thureau-Dangin, F.

o. J. Une relation de la huitième campagne de Sargon, TCL 3, Paris 
